\title{
Activity and Emotion Recognition to Support Early Diagnosis of Psychiatric Diseases
}

\author{
David Tacconi, Oscar Mayora ${ }^{\dagger}$, Paul Lukowicz*, Bert Arnrich, Cornelia Setz, Gerhard Tröster ${ }^{\dagger \dagger}$ and Christian Haring ${ }^{+}$ \\ $\dagger$ CREATE-NET Research Center, Trento, Italy, \\ name. surnamedcreate-net.org \\ * University of Passau, Passau, Germany \\ paul.lukowicz@uni-passau.de \\ †† ETHZ, Zurich, Switzerland \\ \{barnrich, cornelia.setz,troester\}eife.ee.ethz.ch \\ + Psychiatric State Hospital Tirol (PSHT), Innsbruck, Austria \\ christian.haring@tilak.at
}

\begin{abstract}
Diagnosis of psychiatric diseases is currently accomplished with questionnaires filled in by the subjects, usually together with a specialist. Such questionnaires are often based on standard, formal scales, where questions range from the ability to cope with household activities, through social interactions, agitation, level of activity, to quality of sleep. In this paper, we propose a context aware framework to support semi-automation of the diagnosis of such diseases, leveraging on authors experience in the fields of activity and emotion recognition. We also introduce a brief description of the main components defining the architecture of the proposed platform and preliminary work on emotion estimation.

Index Terms-wearable computing, ambient intelligence, psy-
\end{abstract} chiatric questionnaires, mobile devices.

\section{INTRODUCTION}

Depression and severe forms of mental disorders (like the bipolar disorder) are conditions that seriously reduce the quality of life for individuals and their families. Depression is the most common psychiatric disorder and is the leading nonfatal condition globally, while $20 \%$ of the population in western countries suffers from depression during their life time.

Diagnosis of psychiatric diseases such as depression, mania, bipolar disorder and dementia is currently accomplished with questionnaires filled in by the subjects, usually together with a specialist (e.g. a psychotherapist). Such questionnaires are often based on standard, formal scales. Examples include the HAMD scale (used in psychiatry for assessment of depression [1]) the BRMS scale (use for the assessment of mania [2]) or the Blessed Dementia Scale [3]. Typical questions range from the ability to cope with household activities, through social interactions, agitation, level of activity, to quality of sleep.

According to doctors' experience, two problems arise during the evaluation of such questionnaires:

- the items listed in the questionnaires are typically referring to emotional states or activities conducted by the patient during the last weeks or months: the reliability of the answers is typically low, since it strictly depends on the ability of the doctor to understand patient mental health in the last mid and long term period, mainly based on the answers he gives during the session,

- the patient is typically meeting a doctor when he experiences a relapse of the disease; at this point, the doctor can only intervene (often with pharmacotherapy) to reduce the effects of the disease and bring the patient to a more stable situation, but it would be desiarable to visit the patient before, in particular at a moment in time when he can prevent an eventual relapse of a psychiatric disease and avoid severe consequences.

Interestingly, while there is a huge body of work on the application of pervasive computing in the general area of healthcare [4] (including many projects on behavior and life style monitoring), psychotherapy has so far been a neglected area. There have been few works [5] that have been looking at the use of mobile devices to support the patient. As far as the application of activity and context recognition is concerned there has been only very preliminary work [6].

Pervasive computing can represent a great help to doctors and patients in supporting early diagnosis of psychiatric diseases, as described in [7]. In fact, mid to long term behavioral characterization is used in psychiatry, psychology and in conjunction with medical trials. Based on previous experience of the authors, we intend to develop methods for automatic mid to long term behavior characterization that, in terms of the information that they provide, will be comparable to the current practice of standard questionnaires.

In doing so, we concentrate on methods that rely on unobtrusive, easy to deploy sensors and devices, do not need user dependent training, and produce recognition rates adequate for real life applications.

In the following sections, we will show how existing solutions provided by the authors in the fields of activity and emotion recognition can be leveraged and combined to support behavioral recognition. We will present a system architecture that 
can be implemented in difference devices (such as laptops and smartphones) where the various components can be integrated for providing early diagnosis support of psychiatric diseases.

\section{System ARChitecture}

When designing a system architecture for psychiatric diseases patients there are some constraints that have to be considered. First of all, according to doctors' experience, these patients are likely to reject pervasive computing technology in principle: consequently the target devices should be as less obtrusive as possible. For the same reason, patients cannot be asked to perform any training of devices, and this complicates things for emotion and activity recognition.

However, the type of activity and emotion recognition that should be performed has the main advantage of being targeted to medium and long term behavior. In such scenario, higher errors in activity recognition are allowed, since doctors are more interested in average behaviors rather than in instantaneous activity pattern or emotions a patient is feeling in a given moment. In other words, doctors are not interested if a patient is feeling bad one day or he doesn't want to talk to anybody for lunch, but if these behaviors are repeted in time it can be a symptoms of disease's relapse.

The proposed system architecture is shown in Fig. 1. The

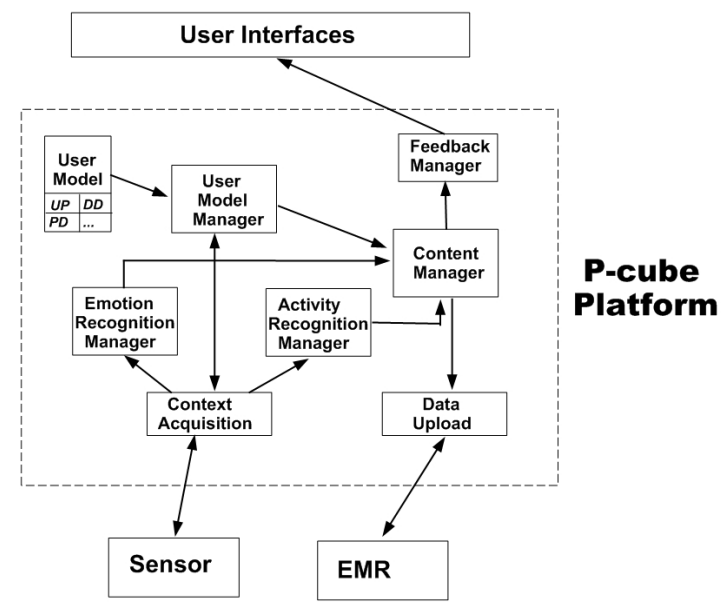

Figure 1. The P-cube platform system architecture

main component is given by the Psychiatric Patient centric Pervasive (P-cube) platform installed on the patient personal device, e.g his smartphones or his laptop (a similar architecture has been implemented by some of the authors in mobile phones, as described in [8]). In the P-cube platform, the User Model includes all patient's characteristics, disease's peculiarities and his preferences. Such information are stored respectively in the User Profile (UP), the Disease Description (DD), the Patient Description (PD). The DD and the PD are filled in by the doctors while the UP is left open for some patient preferences.

P-cube gathers data from the Sensor block through the Context Acquisition module, given the queries of the Emotion Recognition Manager and of Activity Recognition Manager

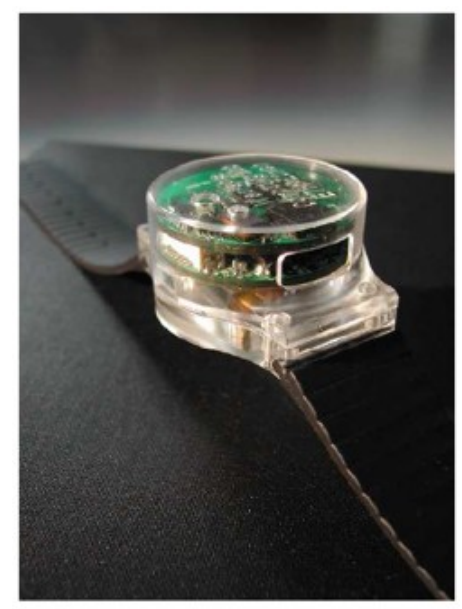

Figure 2. A watch like activitiy recognition platform from [10]

and according to the information provided by the User Model Manager about the patient and his specific disease and condition.

Finally, emotions and activities of interest are managed by the Content Manager, which decides either to provide the patient with some feedbacks through the Feedback Manager and then through the User Interfaces, or store data in the remote EMR (Electronic Medical Records) through the Data Upload module.

Let us now describe how the Emotion Recognition Manager and Activity Recognition Manager (i.e. the central modules of the architecture) can perform their tasks, in particular how behavioural recognition can be achieved using state of the art technology.

\section{A. Activity Recognition}

The main sources of activity information will be a wrist and/or hip worn accelerometer/microphones combination with location information. Indoors we assume that a WLAN access point or BlueTooth nodes placed in key rooms will provide at least room level location. Outdoor we will rely on a mobile phone to provide us with cell location.

In previous experiments we have shown that complex activities can be spotted using such sensor combinations [9]. We have even demonstrated recognition methods running directly on small wrist worn devices (see Fig.2) In addition both indoor and outdoor location will also provide information about daily routine. Indoor patterns of motion will reveal everyday activities such as house cleaning or daily hygiene. Outdoor location can reveal things like shopping, going to work, going out and visiting friends, all of them important aspects in the evaluation of many psychiatric conditions.

\section{B. Emotion Recognition}

In general, spoken messages convey besides information on textual content also non-textual characteristics like intonation, speaking rate or even emotional state. In emotional state classification the emotion that speaker convey in their utterances are 
assessed. For people suffering from mental disease, emotion recognition can provide the therapists with more insights into the daily variations of the patients mental state.

In P-Cube, we started our work on emotion recognition by means of a preliminary feasibility study. To facilitate the development of classification procedures in daily life situations, we restricted ourselves to the recognition of a set of basic emotional classes, e.g. anger, boredom, desperation, disgust, happiness or pride. In the feasibility study, 10 healthy young subjects were asked to express these six emotions in two given sentences with no contextual meaning. The speech data were recorded twice for each emotion using a head set. Adapted from a literature review, a set of appropriate features were identified and extracted from the speech data. Based on these features, a linear discriminant analysis (LDA) was performed. In a leave-one-out cross validation experiment, a classification accuracy of $47.1 \%$ (guessing $16.6 \%$ ) was achieved. In order to compare the result with the human ability of recognizing emotions, a collective of 12 people was provided with the recorded speech data and asked to classify each sentence in one of the six categories. The classification accuracy of $53.0 \%$ was slightly higher than the one obtained with LDA. This provides confidence that an automatic emotional state recognition is a feasible approach. By using more sophisticated classification models, we expect to achieve even better classification results in comparison to human judgers.

\section{Future DiREctions}

In this paper we presented preliminary work defining the main issues in performing activity and emotion recognition for supporting doctors and patients in diagnosing psychiatric disorders, currently recognized only via standard questionnaries. We have also proposed a system architecture designed to preform patients' behavioural monitoring.

Our ongoing work consists in integrating the currently available technologies described in this paper into the platform to perform some laboratory testing. Further field tests will be carried out at the Psychiatric State Hospital in Tirol, together with our medical partners.

\section{REFERENCES}

[1] "The Hamilton Rating Scale for Depression." [Online]. Available: http://healthnet.umassmed.edu/mhealth/HAMD.pdf

[2] "The Bech-Rafaelsen Mania Rating Scale." [Online]. Available: http://www.medalreg.com/www/xdocs/docs_ch18/doc_ch18.19.html

[3] "The Blesseds Dementia Scale." [Online]. Available: http://www.neuropsicol.org/Protocol/blessed.pdf

[4] P. Lukowicz, T. Kirstein, and G. Troster, "Wearable systems for health care applications," Methods Inf. Med, vol. 3, 2004.

[5] P. A. Marco de Sa, Luis Carrico, "Ubiquitous psychotherapy," IEEE Pervasive Computing, vol. 06, no. 1, pp. 20-27, 2007.

[6] T. Westeyn, K. Vadas, X. Bian, T. Starner, and G. D. Abowd, "Recognizing mimicked autistic self-stimulatory behaviors using hmms,' Proceedings of the Ninth IEEE international Symposium on Wearable Computers ISWC, pp. 164-169, 2005.

[7] D. Tacconi, O. Mayora, P. Lukowich, B. Arnrich, G. Troster, and C. Haring, "On the feasibility of using activity recognition and context aware interaction to support early diagnosis of bipolar disorder," Ubicomp 2007 Workshops adjunct proceedings, Ubiwell Workshop, September 2007.
[8] I. Carreras, D. Tacconi, and D. Miorandi, "Data-centric information dissemination in opportunistic environments," Proceedings of IEEE MASS 2007, October 2007.

[9] J. Ward, P. Lukowicz, and G. T. T. Starner, "Activity recognition of assembly tasks using body-worn microphones and accelerometers," IEEE Trans. Pattern Analysis and Machine Intelligence, vol. 28:10, pp. 1553-1567, October 2006.

[10] D. Roggen, N. Bharatula, M. Stäger, P. Lukowicz, and G. Tröster, "From sensors to miniature networked sensor buttons," in Proc. 3rd Int. Conf. on Networked Sensing Systems - INSS 2006, June 2006, pp. 119-122. 